\title{
BIOSSENSOR AMPEROMÉTRICO PARA DETERMINAÇÃO DE PERÓXIDO DE HIDROGÊNIO EM LEITE
}

\author{
Vitor Paulo Andrade da Silva ${ }^{1}$, Carlucio Roberto Alves ${ }^{1}$, Rosa Fireman Dutra ${ }^{2}$, Juliano Elvis de \\ Oliveira ${ }^{3}$, Davide Rondina ${ }^{4}$, Roselayne Ferro Furtado ${ }^{5}$ \\ ${ }^{1}$ Departamento de Química, Universidade Federal do Ceará, CEP 60740-000, Fortaleza-CE. E-mail: \\ vitorvolt@hotmail.com \\ ${ }^{2}$ Departamento de Engenharia Biomédica, Universidade Federal de Pernambuco \\ ${ }^{3}$ Departamento de Ciências e Engenharia de Materiais, Universidade Federal de São Carlos \\ ${ }^{4}$ Departamento de Veterinária, Universidade Estadual do Ceará \\ ${ }^{5}$ Embrapa Agroindústria Tropical, Fortaleza-CE. E-mail: roselayne@cnpat.embrapa.br
}

\section{Resumo:}

Um biossensor amperométrico foi desenvolvido para detecção de peróxido de hidrogênio em amostras de leite. $\mathrm{O}$ biossensor foi construído a partir da imobilização de enzima peroxidase sobre eletrodo impresso de carbono. Parâmetros de otimização visando um melhor desempenho do biossensor foram avaliados. O biossensor apresentou linearidade no intervalo de 5,0 a 40,0 $\mu \mathrm{mol} \mathrm{L}{ }^{-1}$ de $\mathrm{H}_{2} \mathrm{O}_{2}$ em tampão fosfato. Em amostras de leite sem diluição, os limites de detecção e quantificação foram de $0,42 \mu \mathrm{mol} \mathrm{L} \mathrm{L}^{-1}$ e $1,39 \mu \mathrm{mol} \mathrm{L} \mathrm{L}^{-1}$, respectivamente. O biossensor mostrou-se uma alternativa sensível e de baixo custo na detecção de $\mathrm{H}_{2} \mathrm{O}_{2}$ em amostras adulteradas de leite.

Palavras-chave: Peroxidase de raiz forte, eletrodo impresso, glutaraldeído, biossensor

\section{Introdução}


A adição de peróxido de hidrogênio ao leite se reporta ao seu efeito antibacteriano e sua função de dissimulação das más condições higiênico-sanitárias de obtenção, conservação e/ou transporte do leite. Em contato com o leite, a degradação do peróxido de hidrogênio promove a oxidação da espécie química tiocianato $\left(\mathrm{SCN}^{-}\right)$(componente natural do leite) em outra espécie denominada hipotiocianato $\left(\mathrm{SCNO}^{-}\right)$que tem efeito antibacteriano ou bacteriostático [1]. Neste sentido, a adoção dessa prática por produtores objetiva o prolongamento da vida útil do leite por eliminar ou inibir o desenvolvimento de bactérias deterioradoras deste produto.

O método de determinação de peróxido de hidrogênio recomendado pela Legislação brasileira envolve titulação com permanganato de potássio e possui limite de detecção de 300 ppm. A utilização de biossensores possibilitaria a detecção dessa substância com limites de detecção bastante menores, além da praticidade e rapidez das análises. Os biossensores constituem uma alternativa rápida e conveniente para medidas analíticas convencionais no monitoramento de substâncias químicas e bioquímicas e em processos de fermentação e na indústria de alimentos [2-3].

A peroxidase de raiz forte (HRP) é atualmente usada em uma grande variedade de sistemas analíticos e de diagnósticos importantes em laboratórios clínicos, com grande potencial de uso no desenvolvimento de biossensores. A aplicação dessa enzima para determinação de substâncias como a glicose, ácido úrico e colesterol em fluidos biológicos, como sangue, plasma e urina tem sido adotado satisfatoriamente [4].

As enzimas peroxidases podem ser classificadas em relação ao seu grupo prostético (sítio ativo) em hêmicas ou não-hêmicas. Pertencente à classe das hêmicas, citamos a peroxidase de raiz forte, utilizada neste trabalho. As hêmicas apresentam um grupo heme ou ferroprotoporfirina, formado por um íon $\mathrm{Fe}^{3+}$ coordenado a uma protoporfirina IX. Esse grupo catalisa reações de hidrólise envolvendo o íon ferro [5,6,7]. Quando a enzima peroxidase está imobilizada na superfície de um eletrodo, os intermediários oxidados da enzima, Composto-I e Composto-II, podem ser reduzidos para voltarem ao seu estado nativo por dois processos [8] sendo um deles, a transferência de elétrons mediada. Na transferência mediada de elétrons a enzima recebe elétrons de uma substância doadora (mediador). A enzima é então regenerada e o mediador, agora oxidada, é reduzido por elétrons provenientes do eletrodo [9]. No presente trabalho a hidroquinona foi escolhida como mediador eletroquímico do sistema. 
A tecnologia de "screen-printing", conhecida também como "silk-screen", tem sido empregada com sucesso na fabricação de eletrodos [10]. Esta técnica possibilita a produção em larga escala de eletrodos a um baixo custo, com elevada reprodutibilidade, o que garante um potencial de automação com ótimo custo-benefício [11]. Outra vantagem do uso dessa tecnologia é a possibilidade de construção de eletrodos descartáveis.

Nesse estudo, o eletrodo de trabalho consistiu de um eletrodo impresso com tinta comercial condutora de carbono. As tintas de carbono são compostas basicamente por partículas de grafite, solvente e um agente aglutinante. Na aplicação de sensores, são importantes, pois geram baixas correntes de fundo e permitem trabalho em ampla janela de potencial $[12,13]$.

A etapa de incorporação do biocomponente ao eletrodo é crucial no desenvolvimento de um biossensor [14]. A imobilização pode afetar a conformação da enzima, e assim sua estabilidade, ou fazer com que os sítios ativos se tornem menos acessíveis para a difusão de substratos. Sendo assim, o método de imobilização deve ser compatível com a biomolécula que está sendo imobilizada, com a superfície do sensor ou matriz na qual está sendo imobilizada e com o meio onde o sensor será utilizado. Nesse trabalho foi utilizado o glutaraldeído, um reagente bifuncional, para a imobilização da enzima à superfície do eletrodo, onde, um grupo aldeído de uma extremidade da molécula se ligou ao eletrodo e o outro grupo se ligou à enzima.

O objetivo deste trabalho foi desenvolver um biossensor amperométrico descartável de baixo custo para a detecção de peróxido de hidrogênio em amostras de leite. O desempenho do biossensor foi avaliado em amostras de tampão contendo peróxido de hidrogênio e em amostras de leite adulterado previamente no laboratório com peróxido de hidrogênio, no intuito de melhor estabelecer comparações entre o funcionamento do mesmo em condições experimentais e práticas.

\section{Materiais e métodos}

\subsection{Reagentes}

Cloreto de potássio foi adquirido da Reagen ${ }^{\circledR}$ e o cloreto de sódio da Dinâmica ${ }^{\circledR}$. O hexacianoferrato(III) de potássio, o glutaraldeído $25 \%(\mathrm{~m} / \mathrm{v})$, a hidroquinona e o peróxido de hidrogênio $30 \%(\mathrm{~m} / \mathrm{v})$ foram obtidos da Vetec ${ }^{\circledR}$. A enzima peroxidase de horseradish - Sigma Type VI foi adquirida da Sigma Aldrich ${ }^{\circledR}$. O nitrogênio utilizado para a remoção do oxigênio do 
eletrólito suporte foi conseguido da White Martins ${ }^{\circledR}$ com grau de pureza de 99,9\%. A tinta condutora de carbono utilizada na construção do eletrodo impresso foi adquirida da Eletrodag ${ }^{\circledR}$. A folha de acetato que serviu como suporte do eletrodo impresso era da marca Apollo ${ }^{\circledR}$. Todos os outros reagentes químicos utilizados foram de grau analítico. As soluções tampão fosfato que foram utilizadas como eletrólito suporte foram obtidas pela mistura das soluções de $\mathrm{Na}_{2} \mathrm{HPO}_{4} 0,1$ mol L ${ }^{-1}$ e de $\mathrm{NaH}_{2} \mathrm{PO}_{4} 0,1 \mathrm{~mol} \mathrm{~L}^{-1}$. Todas as soluções foram preparadas com água deionizada.

\subsection{Preparação dos eletrodos impressos}

A impressão dos eletrodos foi feita com a deposição da tinta condutora de carbono Eletrodag ${ }^{\circledR}$ sobre a superfície de folha de acetato pelo método "casting" no intuito de simular a técnica industrial de "screen-printing". Nesse método, a tinta é forçada a passar através de uma tela ou molde vazado para ser depositada sobre um substrato plano. O desenho, definido pelas partes abertas da tela, é reproduzido no substrato.

Os eletrodos foram feitos com área geométrica de $0,78 \mathrm{~cm}^{2}$ e largura da trilha igual a 2,0 mm. Após o processo de impressão, foram levados à estufa a $80^{\circ} \mathrm{C}$ por uma hora para a secagem da tinta. Em seguida, aplicou-se a eles uma camada parcial de adesivo de PVC que definiu a área para ser a superfície do eletrodo e a área a ser usada como contato elétrico.

Os eletrodos impressos (EI) foram submetidos a uma série de 10 ciclos em voltametria cíclica com potenciais de inversão de $-600 \mathrm{mV}$ e $+600 \mathrm{mV}$ a velocidade de varredura igual a $100 \mathrm{mV} \mathrm{s}^{-1}$ em tampão fosfato $0,1 \mathrm{~mol} \mathrm{~L}^{-1} \mathrm{pH}$ 6,0. De acordo com BODOKI et al (2008) [15], esse procedimento contribui também para uma diminuição da hidrofobicidade da superfície.

\subsection{Preparação do biossensor}

Os EI foram imersos durante uma hora em solução de glutaraldeído (GA) 2,5\% (m/v) e, posteriormente imersos por uma hora em solução de enzima peroxidase de raiz forte (HRP) $1 \mathrm{mg}$ $\mathrm{mL}^{-1}$. A solução de HRP consistia de HRP solubilizada em tampão fosfato $0,1 \mathrm{M} \mathrm{pH} \mathrm{6,5.} \mathrm{Ao}$ final de cada procedimento os EI foram lavados com água deionizada para a remoção de excessos e moléculas fracamente adsorvidas. 


\subsection{Instrumentação}

Uma célula eletroquímica de $10 \mathrm{~mL}$ com eletrodo auxiliar de platina, eletrodo de referência de $\mathrm{Ag} / \mathrm{AgCl}$ e eletrodos impressos foi utilizada para as medidas voltamétricas e amperométricas em Potenciostato/Galvanostato Autolab PGSTAT302N. As soluções foram purgadas com nitrogênio por 10 minutos antes de cada ensaio.

\subsection{Caracterização e otimização da resposta do biossensor}

Os parâmetros de resposta do biossensor EI-GA-HRP estudados foram o efeito do potencial de trabalho, efeito do $\mathrm{pH}$, concentração do mediador, concentração enzimática, estabilidade do biossensor e caracterização superficial do biossensor.

\subsection{Caracterização superficial do biossensor}

A caracterização microscópica da superfície dos eletrodos impressos e da superfície do biossensor foi realizada mediante emprego de um microscópio eletrônico de varredura ZEISS modelo DSM-960, operando com aceleração de voltagem de 5kV.

\subsection{Validação do biossensor em amostras de leite comercial longa vida (UHT)}

A validação do biossensor foi realizada em amostras de leite comercial longa vida (UHT), diluídas na proporção de 1:100, 1:10 e 1:2, e em leite não diluído. As diluições foram feitas em tampão fosfato $0,1 \mathrm{~mol} \mathrm{~L}^{-1} \mathrm{pH} 6,5$ e adicionado de hidroquinona $30 \mu \mathrm{mol} \mathrm{L}^{-1}\left(\Delta \mathrm{E}_{\text {apl }}=-\right.$ $250 \mathrm{mV}$ vs. $\mathrm{Ag} / \mathrm{AgCl})$.

Os intervalos de confiança para os limites de detecção e de quantificação do biossensor foi calculado utilizando o teste $t$ de Student com nível de confiança de $95 \%$.

\section{Resultados}

\subsection{Preparação e otimização do biossensor}

Para preparar a solução de enzima, foi escolhido o tampão fosfato, pois, dentre os analisados, este proporcionou melhor resposta para o sinal do biossensor na presença de peróxido de hidrogênio utilizando HRP nos trabalhos desenvolvidos por Rosatto (2001) e [7] Mendes 
(2008) [16]. O pH da solução tampão utilizada nos ensaios iniciais foi 6,0 , valor ideal para a HRP segundo recomendações do distribuidor da enzima (Sigma Aldrich ${ }^{\circledR}$ ).

A Figura 1 indica as curvas voltamétricas obtidas para o EI, EI-GA e EI-GA-HRP, em tampão fosfato $0,1 \mathrm{~mol} \mathrm{~L} \mathrm{~L}^{-1}$ contendo hidroquinona $25 \mu \mathrm{mol} \mathrm{L} \mathrm{L}^{-1}$ e $\mathrm{H}_{2} \mathrm{O}_{2} 80 \mu \mathrm{mol} \mathrm{L} \mathrm{L}^{-1}$ com velocidade de varredura igual a $50 \mathrm{mV} \mathrm{s}^{-1}$. Podemos observar que EI e EI-GA possuem curvas com perfis semelhantes, com picos de redução e oxidação para a hidroquinona em torno de -100 e $500 \mathrm{mV}$, respectivamente. Uma curva bastante característica para o biossensor EI-GA-HRP também pode ser vista. $\mathrm{O}$ pico em torno de $-250 \mathrm{mV}$ estaria relacionado à redução eletroquímica da hidroquinona que foi oxidada no processo de regeneração da HRP [9, 17-18].

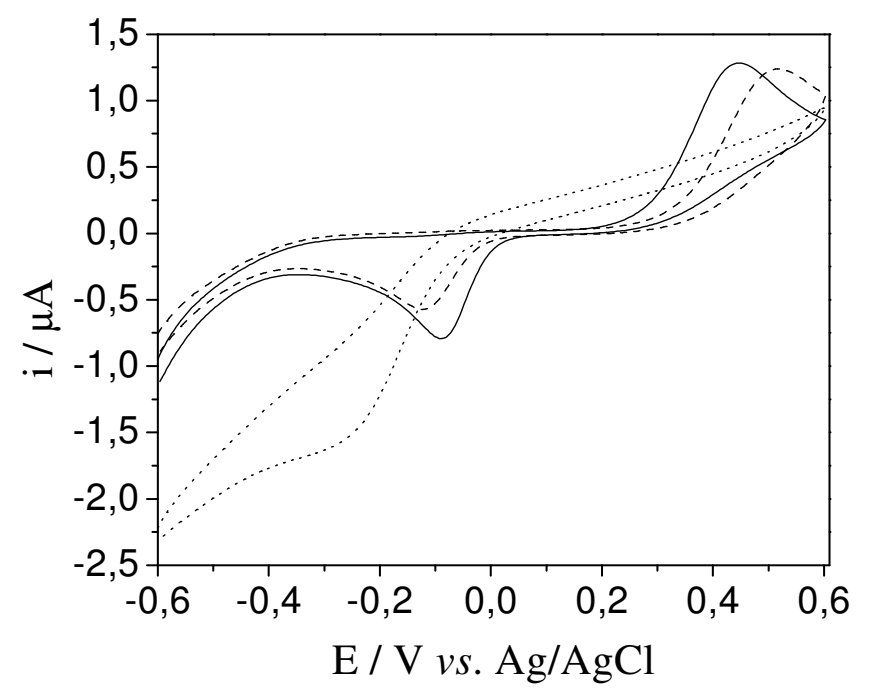

Figura 1 - Curvas voltamétricas para EI (--), EI-GA (---) e EI-GA-HRP (….) em tampão fosfato $0,1 \mathrm{~mol} \mathrm{~L}^{-1} \mathrm{pH} 6,0$ contendo hidroquinona $25 \mu \mathrm{mol} \mathrm{L} \mathrm{L}^{-1}$ como mediador e $80 \mu \mathrm{mol} \mathrm{L} \mathrm{L}^{-1} \mathrm{de}$ peróxido de hidrogênio. Velocidade de varredura: $50 \mathrm{mV} \mathrm{s}^{-1}$.

Ensaios de cronoamperometria foram realizados para avaliar a influência do potencial aplicado, efeito do $\mathrm{pH}$, concentração do mediador e concentração enzimática na resposta do biossensor. A Figura $2 \mathrm{a}$ indica que a corrente de redução aumentou à medida que o potencial variou de -100 a $-250 \mathrm{mV}$, a partir de onde praticamente não se altera. Então, o potencial selecionado para os ensaios subseqüentes foi $-250 \mathrm{mV}$. Potenciais relativamente altos devem ser evitados para diminuir a probabilidade de outras espécies químicas indesejadas em solução sofrerem processo oxirredutivo, e assim, prejudicarem a leitura eletroquímica. O 
peróxido de hidrogênio pode ser oxidado em eletrodo de platina ou carbono a valores de potencial entre 300 e $600 \mathrm{mV}$ [19]. Dessa forma, a utilização de mediadores eletroquímicos é vantajosa, pois o potencial de trabalho do eletrodo enzimático é determinado pelo potencial de oxirredução do mediador. CAMACHO et al (2007) [20], fazendo experimentos com peroxidase imobilizada em eletrodo de ouro modificado com $\beta$-ciclodextrina e utilizado hidroquinona como mediador, definiu em seu estudo $-250 \mathrm{mV}$ vs. $\mathrm{Ag} / \mathrm{AgCl}$ como potencial de trabalho mais apropriado.

$\mathrm{O}$ pH do meio afeta os grupos ionizáveis da estrutura enzimática, por isso, a resposta eletrocatalítica do biossensor enzimático é bastante influenciada pela variação de pH. A Figura $2 \mathrm{~b}$ indica um aumento na corrente de redução com o aumento do $\mathrm{pH}$, sendo máximo em 6,5. $\mathrm{O}$ $\mathrm{pH}$ de 6,5 foi adotado para os estudos posteriores, sendo este também próximo ao $\mathrm{pH}$ natural do leite.

A Figura $2 \mathrm{c}$ indica a influência da concentração do mediador hidroquinona na resposta do biossensor. Há um aumento de corrente catódica com o aumento da concentração de hidroquinona de 5 a $25 \mu \mathrm{mol} \mathrm{L} \mathrm{L}^{-1}$ e acima desse valor, torna-se praticamente constante. Isto ocorre, porque em baixas concentrações de hidroquinona, a resposta do biossensor depende da concentração do mediador (mediador-dependente) [16].

A avaliação da resposta amperométrica do biossensor em função da concentração de enzima HRP é demonstrada na Figura 2d. Observou-se um incremento significativo de corrente quando a concentração de enzima é aumentada de 0,5 para $1,0 \mathrm{mg} \mathrm{mL}^{-1}$. A partir de $1,0 \mathrm{mg} \mathrm{mL}^{-1}$ até $2,0 \mathrm{mg} \mathrm{mL}^{-1}$ há pouca variação de corrente. Sendo assim, a concentração de $1,0 \mathrm{mg} \mathrm{mL}^{-1}$ foi considerada satisfatória, sendo utilizada nos ensaios posteriores.
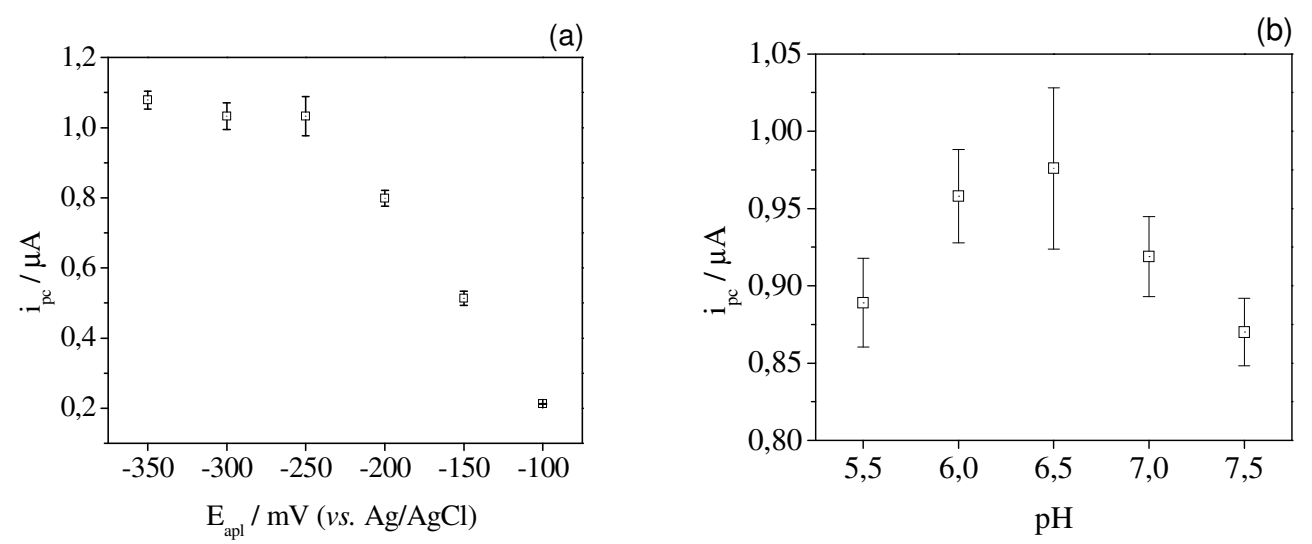

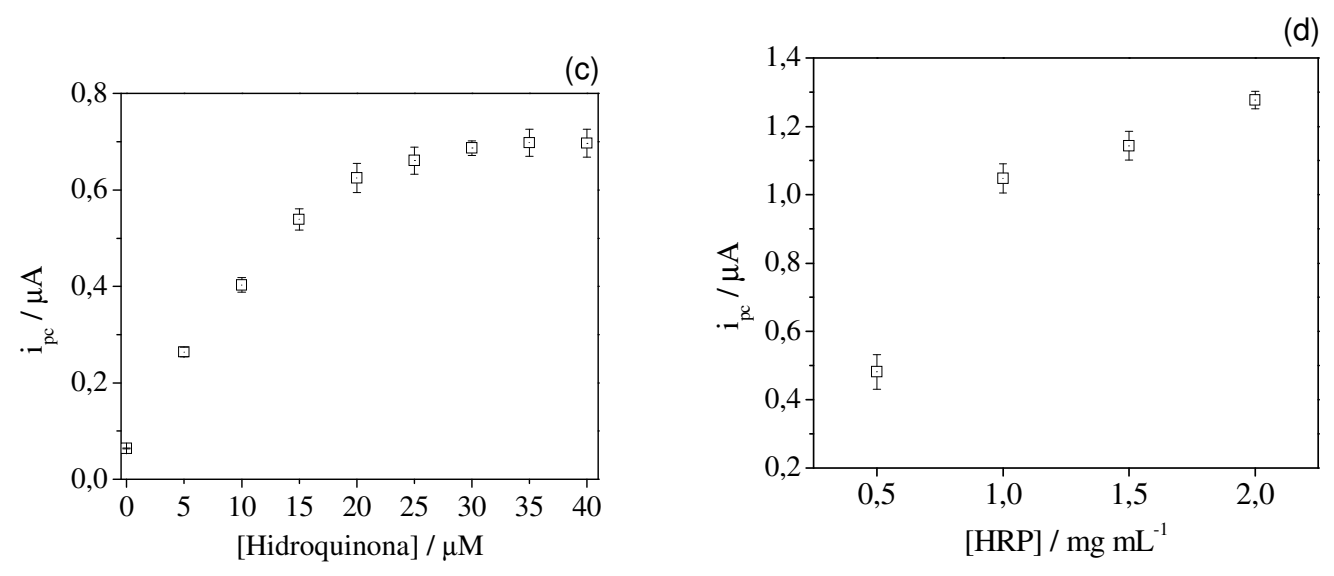

Figura 2 - Ensaios cronoamperométricos em tampão fosfato $0,1 \mathrm{~mol} \mathrm{~L}^{-1} \mathrm{pH} 6,5$ na presença de hidroquinona $25 \mu \mathrm{mol} \mathrm{L} \mathrm{L}^{-1} \mathrm{H}_{2} \mathrm{O}_{2} 100 \mu \mathrm{mol} \mathrm{L} \mathrm{L}^{-1}$ para determinação do efeito do potencial (a), influência do pH (b), Influência da concentração do mediador hidroquinona (c), influência da concentração da enzima HRP (d) na resposta do biossensor.

\subsection{Estabilidade do biossensor}

O biossensor mostrou boa estabilidade em 50 ciclos consecutivos de varredura (Figura 3). Após o primeiro ciclo, verificou-se que 96,3\% da resposta inicial do biossensor foi mantida. Ao final de 50 ciclos, a resposta correspondeu a 90,3\% da inicial. A perda de resposta após o primeiro ciclo de varredura (cerca de 3,7\%) indica que forças de adsorção física podem estar envolvidas do processo de imobilização enzimática na superfície do eletrodo, uma vez que essas forças são de natureza fraca e, durante o processo de varredura, uma pequena parte da enzima reticulada tenha sido lixiviada para a solução. 


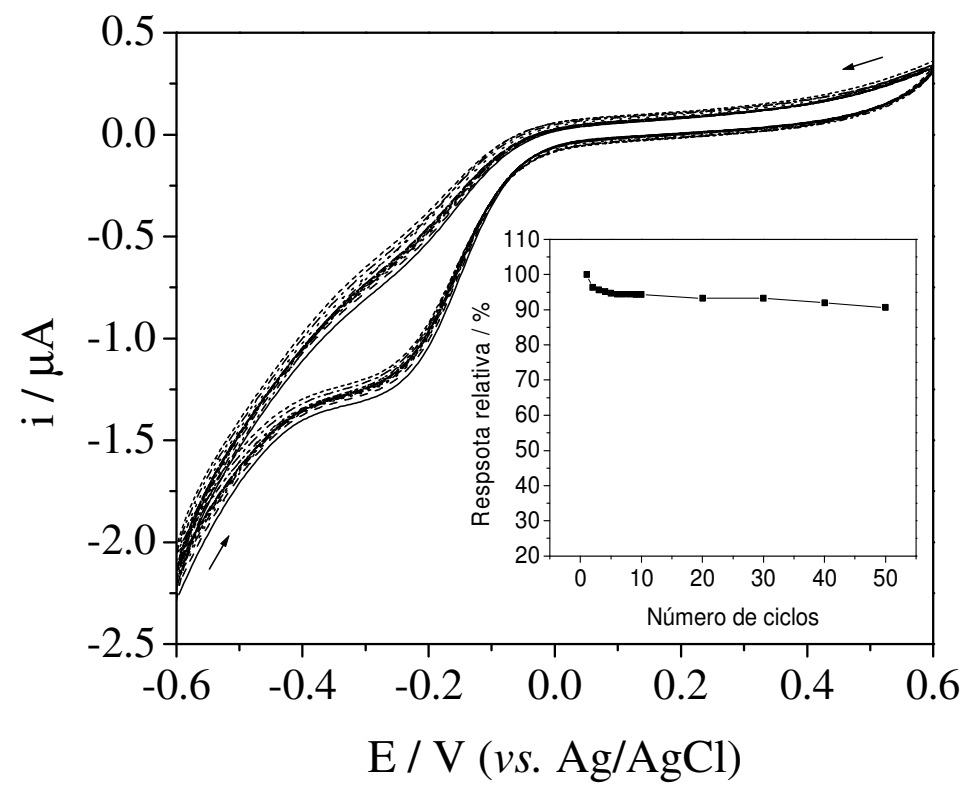

Figura 3 - Estabilidade do biossensor avaliada em tampão pH 6,5 na presença hidroquinona 25 $\mu \mathrm{mol} \mathrm{L} \mathrm{L}^{-1}$ e $\mathrm{H}_{2} \mathrm{O}_{2} 100 \mu \mathrm{mol} \mathrm{L} \mathrm{L}^{-1}$ usando voltametria cíclica (50 ciclos) com potenciais de inversão de -600 a $600 \mathrm{mV}$ vs. Ag/AgCl. Velocidade de varredura: $50 \mathrm{mV} \mathrm{s}^{-1}$.

\subsection{Caracterização da superfície do biossensor}

A análise da superfície por MEV mostrou que os eletrodos impressos apresentaram estruturas rugosas de partículas de carbono (Figura 4a). Estas estruturas rugosas promovem um aumento da área superficial do eletrodo contribuindo para o aumento do número de sítios ativos na superfície de eletrodo, que por sua vez, contribuem para um aumento da cobertura de HRP. Sendo assim, uma maior quantidade de $\mathrm{H}_{2} \mathrm{O}_{2}$ pode ser catalisada pela HRP. A Figura $4 \mathrm{~b}$ mostra a superfície do eletrodo modificado. Há uma grande redução no número de sulcos na superfície do eletrodo impresso indicando que eles foram preenchidos de forma homogênea durante a reticulação do glutaraldeído com a HRP. 

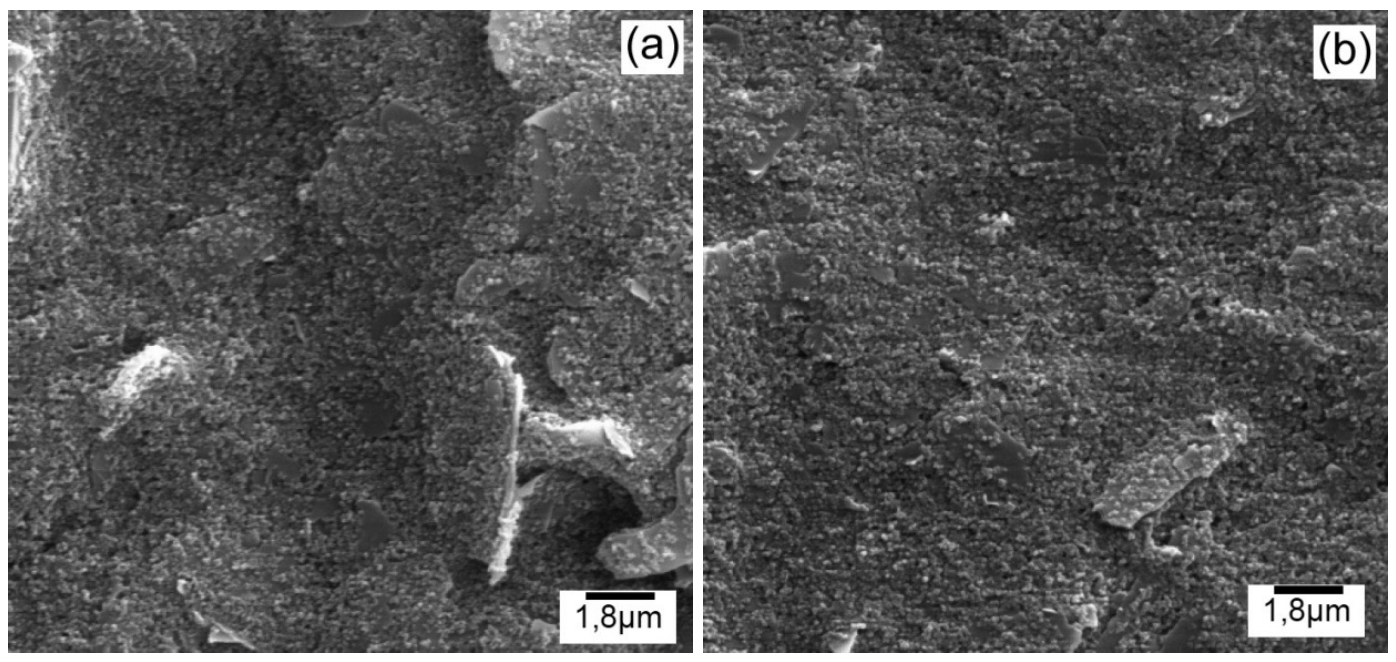

Figura 4 - Micrografia por MEV do EI (ampliação de 5000 x). (a) sem imobilização e (b) com imobilização de GA e HRP.

\subsection{Curva analítica}

A curva analítica do biossensor em tampão fosfato e em amostras de leite (Figura 5) foi construída a partir da adição de $\mathrm{H}_{2} \mathrm{O}_{2}$, utilizando os valores otimizados dos parâmetros operacionais selecionados para o estudo.

Em tampão, a curva apresentou boa linearidade no intervalo de concentração 5,0 a $60,0 \mu \mathrm{mol} \mathrm{L} \mathrm{L}^{-1}$ de $\mathrm{H}_{2} \mathrm{O}_{2}$, com coeficiente de linearidade $\left(\mathrm{R}^{2}\right)=0,99$. O limite de detecção (LD) foi calculado como sendo três vezes o desvio padrão do branco dividido pelo coeficiente angular da reta, e o de quantificação (LQ) como sendo dez vezes o desvio padrão do branco dividido pelo coeficiente angular da reta. Os valores para os limites de detecção e quantificação encontrados foram de $0,41 \mu \mathrm{mol} \mathrm{L}{ }^{-1}$ e $1,4 \mu \mathrm{mol} \mathrm{L}^{-1}$, respectivamente.

Em amostras de leite com e sem diluição, o biossensor apresentou linearidade no intervalo de 5,0 a 40,0 $\mu \mathrm{mol} \mathrm{L}{ }^{-1}$ de $\mathrm{H}_{2} \mathrm{O}_{2}$, apesar da grande ocorrência de analitos interferentes no leite (ácidos graxos, proteínas, vitaminas e outros). Em amostras de leite sem diluição, o limite de detecção para $\mathrm{H}_{2} \mathrm{O}_{2}$ foi de $0,42 \mu \mathrm{mol} \mathrm{L} \mathrm{L}^{-1}$ e o de quantificação foi de $1,39 \mu \mathrm{mol} \mathrm{L^{-1 }}$ bastante similar aos encontrados para as análises em tampão. 


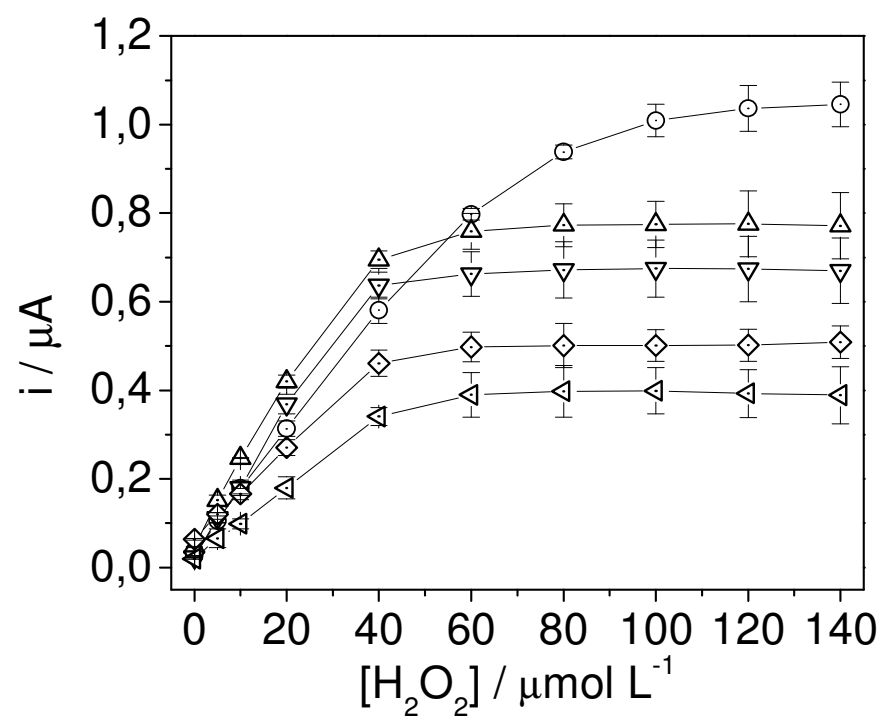

Figura 5 - Curva analítica do biossensor na detecção de peróxido de hidrogênio em: tampão fosfato $0,1 \mathrm{~mol} \mathrm{~L}^{-1} \mathrm{pH} 6,5(\mathrm{O})$, leite com diluição de 1:100 ( $\left.\square\right), 1: 10(\nabla), 1: 2(\diamond)$ e sem diluição $(\triangleleft)$. Todos adicionados de hidroquinona $30 \mu \mathrm{mol} \mathrm{L}^{-1}, \Delta \mathrm{E}_{\mathrm{apl}}=-250 \mathrm{mV} v s . \mathrm{Ag} / \mathrm{AgCl}$.

Podem ser observados a partir da Figura 6, os intervalos de confiança para os limites de detecção e de quantificação, respectivamente, para o biossensor EI-GA-HRP em tampão fosfato $0,1 \mathrm{~mol} \mathrm{~L}^{-1}$, leite nas diluições de 1:100, 1:10, 1:2 e sem diluição (adicionado de hidroquinona $30 \mu \mathrm{mol} \mathrm{L}{ }^{-1}, \Delta \mathrm{E}_{\text {apl }}=-250 \mathrm{mV} v$ s. $\mathrm{Ag} / \mathrm{AgCl}$ ). $\mathrm{O}$ teste $t$ de Student foi usado para o cálculo dos limites de confiança com nível de confiança igual a $95 \%$. 
(a)

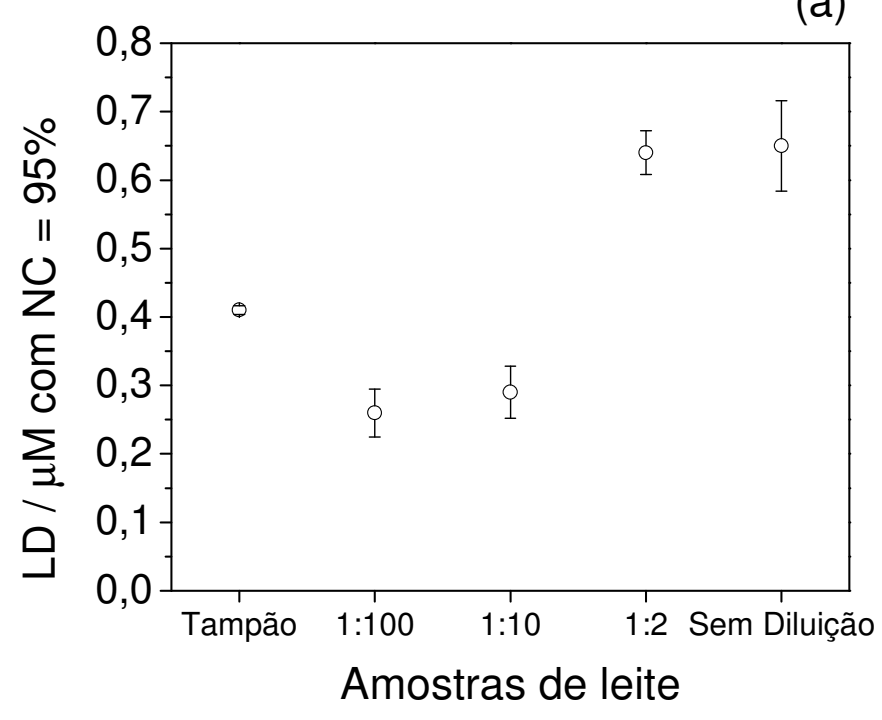

(b)

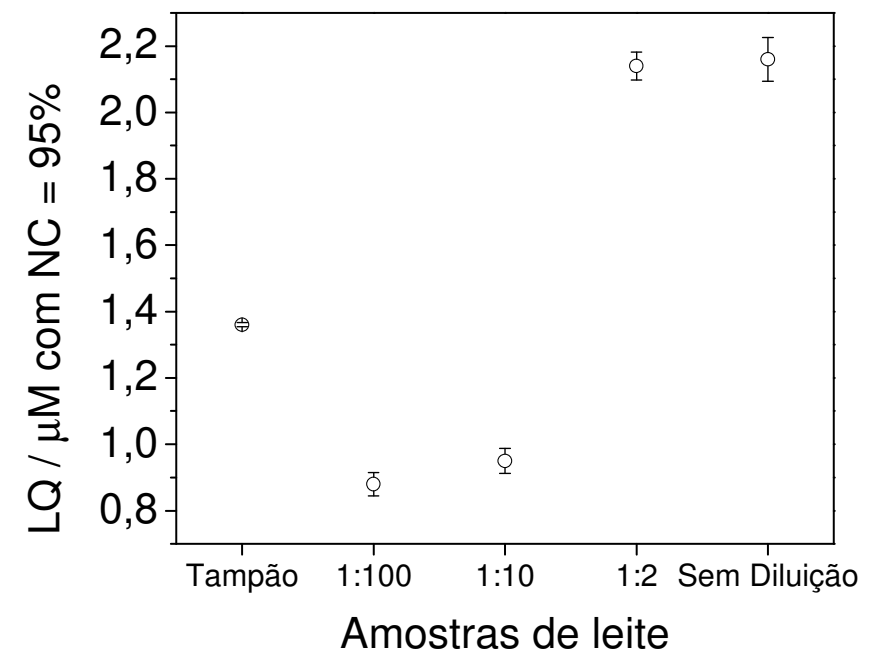

Figura 6 - Intervalo de confiança para os limites de detecção (a) e de quantificação (b) do biossensor para o $\mathrm{H}_{2} \mathrm{O}_{2}$ em tampão fosfato $0,1 \mathrm{~mol} \mathrm{~L}^{-1} \mathrm{pH}$ 6,5, leite com diluição de 1:100, 1:10, 1:2 e sem diluição, calculado a partir de nível de confiança igual a 95\%.

Os resultados para os limites de detecção e quantificação considerando intervalo de confiança igual a 95\%, indicam que não há diferença significativa entre amostras de leite diluídas a 1:100 e 1:10. Da mesma forma, não há diferença significativa para as amostras de leite diluído 1:2 e sem diluição. Sendo assim, recomendamos o uso do biossensor a partir da amostra 
sem diluição e caso alcance valores que saturem a linearidade da curva analítica, existindo a intenção de quantificar o analito em questão, seja feita a diluição da amostra em tampão fosfato na proporção de 1:10.

A equação de Michaelis-Menten é usada para se calcular a atividade catalítica de uma enzima, e pode ser algebricamente rearranjada para a Equação 1, chamada LineweaverBurk, que relaciona valores de corrente e concentração de substrato,

$\frac{1}{I_{s}}=\frac{K_{m}}{I_{m a x}} \cdot \frac{1}{c}+\frac{1}{I_{m a x}}$

onde $\mathrm{I}_{\mathrm{s}}$ é corrente de estado estacionário após a adição de $\mathrm{H}_{2} \mathrm{O}_{2}$, $\mathrm{I}_{\text {máx }}$ é corrente máxima, obtida após a saturação da curva, $\mathrm{K}_{\mathrm{m}}$ é a constante de Michaelis-Menten, c é a concentração de $\mathrm{H}_{2} \mathrm{O}_{2}$ na solução. Baseado na curva analítica obtida na resposta do biossensor em solução tampão, construiu-se um gráfico considerando-se $1 / \mathrm{I}_{\mathrm{pc}}$ em função de $1 / \mathrm{c}$, onde foi obtida uma reta com inclinação positiva de coeficiente angular igual a $\mathrm{K}_{\mathrm{m}} / \mathrm{I}_{\text {Máx }}$ com intercepto em $1 / \mathrm{I}_{\text {Máx }}$ (Figura 25 ). A partir da Figura 25 e utilizando-se a Equação 10, obteve-se $\mathrm{K}_{\mathrm{m}} \cong 48 \mu \mathrm{M}$, considerado, quando comparado na literatura, um valor de alta capacidade catalítica [21].

\section{Conclusões}

O biossensor apresentado neste trabalho é de fácil construção e bastante sensível na detecção de peróxido de hidrogênio em amostras de leite sem diluição.

Esse dispositivo mostrou ser uma ferramenta útil na análise de adulteração de leite por peróxido de hidrogênio, representando uma alternativa promissora para os atuais métodos de análise recomendados pela Anvisa.

\section{Agradecimentos}

Os autores agradecem ao CNPq, Embrapa e Capes pelo suporte financeiro. 


\section{Referências}

[1] T. M. Aune, E. L. Thomas, European Journal of Biochemistry. 88 (1977) 209.

[2] L. Yang, W. Wei, X. Gao, J. Xia, H. Tao, Talanta. 68 (2005) 40.

[3] A. R. Harris, J. Zhang, A. Konash, D. Elton, M. Hyland, A. M. Bond, J Solid State Electrochem, 12 (2008) 1301.

[4] A. Pandey, C. Webb, C. R. Socool, C. Larroche, Enzime Technology, Springer Science, New York, 2006.

[5] B. Plietker, Iron catalysis in organic chemistry: reactions and applications, Wiley-VCH, Weinheim, 2008.

[6] H. B. Dunford, Peroxidases e catalases: biochemistry, biophysics, biotechnology and physiology, John Wiley \& Sons, New Jersey, v. 2, 2010.

[7] S. S. Rosatto, R. S. Freire, N. Durán, L. T. Kubota, Química Nova. 24 (2001) 77.

[8] T. Ruzgas, E. Csöregi, J. Emnéus, L. Gorton, G. Marko-Varga, Analytical Chimica Acta. 330 (1996) 123.

[9] C. V Uliana, C. S. Riccardi, H. Yamanaka, Eclética Química. 33 (2008) 67.

[10] L. Tymecki, S. Glab, R. Koncki, Sensors. 6 (2006) 390.

[11] V. B. Nascimento, L. Angnes, Química Nova. 21 (1998) 614.

[12] D. Craston, C. Jones, D. Williams, N. El Murr, Talanta. 38 (1991) 17.

[13] J. Wang, Electrochimica Acta. 43 (1998) 3459.

[14] S. Cosnier, Analytical Bioanalytical Chemistry. 377 (2003) 507.

[15] E. Bodoki, S. Laschi, I. Palchetti, R. Sandulescu, M. Mascini, Talanta. 76 (2008) 288.

[16] R.K. Mendes, R.F. Carvalhal, L.T. Kubota, Journal of Electroanalytical Chemistry. 612 (2008) 164.

[17] I. R. W. Z. Oliveira, I. C. V. Vieira, Quimica Nova. 29 (2006) 932.

[18] A. Chaubey, B. D. Malhotra, Biosensor and Bioelectronics. 17 (2002) 441.

[19] G. J. Moody, G. S. Sanghera, J. D. R. Thomas, The Analisty. 111 (1986) 605.

[20] C. Camacho, J. C. Matías, B. Chico, R. Cao, L. Gómez, B. K. Simpson, R. Villalonga, Electroanalysis, 19 (2007) 2538.

[21] K. I. Ozoemena, T. Nyokong, Electrochimica Acta. 51 (2006) 5131. 


\begin{abstract}
An amperometric biosensor was developed for detection of hydrogen peroxide in milk samples. The biosensor was constructed from the immobilization of peroxidase on printed carbon electrode. Optimization parameters were evaluated in order to obtain better performance of the biosensor. The biosensor showed linearity in the range 5.0 to $40.0 \mu \mathrm{mol} \mathrm{L}{ }^{-1} \mathrm{H}_{2} \mathrm{O}_{2}$ in phosphate buffer. Milk samples without dilution reported the detection limit for $\mathrm{H}_{2} \mathrm{O}_{2}$ of 0.42 $\mu \mathrm{mol} \mathrm{L}{ }^{-1}$ and quantification of $1.39 \mu \mathrm{mol} \mathrm{L}^{-1}$. The biosensor could be a sensitive and inexpensive alternative for the detection of $\mathrm{H}_{2} \mathrm{O}_{2}$ in adulterated milk samples.
\end{abstract}

Keywords: Peroxidase radish, screen printed, glutaraldehyde, biosensor 